\title{
Nucleolar-Associated Chromatin
}

National Cancer Institute

\section{Source}

National Cancer Institute. Nucleolar-Associated Chromatin. NCI Thesaurus. Code C33181.

Heterochromatin that is associated with the nucleolus. 